\title{
Allergieprävention: Hochallergene Nahrungsmittel füttern, aber wann?
}

— Die aktuelle Leitlinie zur Allergieprävention empfiehlt, Beikost nach Vollendung des vierten Lebensmonats einzuführen. Eine vorsorgliche Meidung potenter Nahrungsmittelallergene im ersten Lebensjahr sollte nicht erfolgen, da der präventive Effekt einer solchen Restriktion nicht gesichert sei [Schäfer T et al. Allergo J Int 2014;23:186-99]. Die Leitlinie nimmt jedoch keine Stellung zu der Frage, ob das Füttern hochallergener Speisen vor einem Alter von zwölf Monaten sogar dazu beitragen könnte, Allergien vorzubeugen, sagte Prof. Dr. Kirsten Beyer vom Kinderallergologischen Studienzentrum an der Charité Berlin.

Viel Aufmerksamkeit erhielt eine kürzlich publizierte Interventionsstudie aus Großbritannien, die dies für die Erdnussallergie untersuchte: Die Studie belegt, dass bei Kindern mit schwerer Neurodermitis und/ oder Hühnereiweißallergie die Gabe von Erdnuss ab einem Alter von vier bis elf Mo- naten das Risiko, später eine Erdnussallergie zu entwickeln, signifikant reduziert [Du Toit G et al. N Engl J Med 2015;26;372:803-13]. In einer placebokontrollierten Studie an der Charité Berlin wurde überprüft, ob ein solcher präventiver Effekt auch bei der Gabe von Hühnerei ab dem Alter von vier bis sechs Lebensmonaten zu erwarten ist. Testungen vor Beginn der Intervention zeigten, dass bereits in diesem Alter 23 der 406 Kinder gegen Hühnerei sensibilisiert waren, berichtete Beyer. Die nicht sensibilisierten Kinder erhielten randomisiert entweder eine Hühnerei-freie Ernährung oder dreimal pro Woche Hühnereipulver. In der Placebogruppe entwickelten vier, in der Verumgruppe acht Kinder bis zum Alter von zwölf Monaten eine Sensibilisierung gegen Hühnerei. Die Einführung von Hühnerei in die Beikost im ersten Lebensjahr war somit keine erfolgreiche Präventionsstrategie, so Beyer. Die Prävention

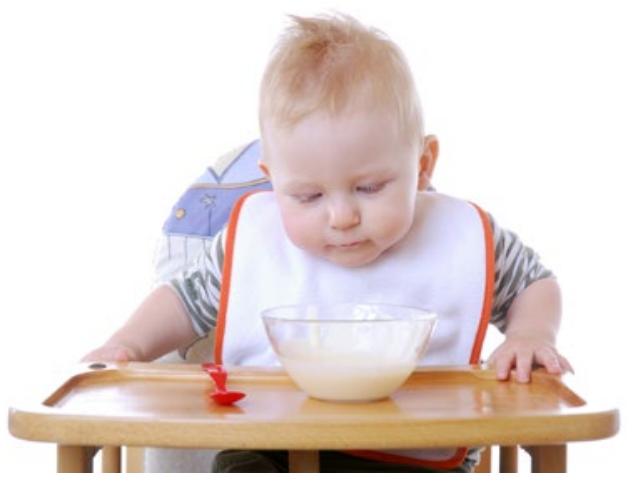

müsse hier möglicherweise schon vor Ende des vierten Lebensmonats ansetzen. Mit Spannung erwartet werden nun die 웅 Ergebnisse der EAT (Enquiring About Tolerance)-Studie (www.eatstudy.co.uk), die der $\stackrel{\frac{\pi}{0}}{0}$ Frage nachgeht, ob das Zufüttern von Kuhmilch, Hühnerei, Fisch, Weizen, Sesam und Erdnuss schon ab einem Alter von drei Monaten die spätere Prävalenz von Nahrungsmittelallergien senken kann.

Angelika Bauer-Delto

INA-Symposium: Die neue Leitlinie Allergieprävention - schon wieder veraltet? Ein Statement zur Einführung "hochallergener" Nahrungsmittel

\section{Allergisches Kontaktekzem - die üblichen Verdächtigen}

- Bei einer Lebenszeitprävalenz in der Größenordnung von $15 \%$ zählt das allergische Kontaktekzem mit zu den häufigsten und gleichzeitig auch zu den am häufigsten vermeidbaren Spielarten der Allergie. Gäbe es da nicht ein Problem. „Die Gesundheits-



politik hat auf diesem Sektor auf ganzer Linie versagt ", befand Prof. Dr. Thomas Fuchs, Universitätsklinik Göttingen. Als irritierend empfindet es der Dermatologe, dass man es seit Jahrzehnten immer mit den gleichen Kontaktallergenen zu tun hat - an der Spitze mit Nickelsulfat, Duftstoff-Mixen, Perubalsam, Kobaltchlorid oder Kaliumdichromat. Irritierend deshalb, weil man etwa am Beispiel der Chromatallergie sehen kann, was man durch geeignete Maßnahmen, wie eine Absenkung des Chromatgehaltes in Zement, alles erreichen kann. In diesem Fall einen signifikanten Rückgang der Neusensibilisierungen bei Männern. Viele andere wissenschaftlich fundierte Daten würden jedoch schlichtweg ignoriert, wunderte sich Fuchs. "Die Botschaft der Diagnose Kontaktallergie ist simpel und eindeutig und lautet Allergenkarenz", so Fuchs. Als Adressaten dieser Botschaft nannte Fuchs insbesondere die Industrie, politische Institutionen und die entsprechenden Überwachungsbehörden. Vermittelt werden müsse vor allem die Er- kenntnis, dass die Erkrankung gewissermaßen hausgemacht ist und nicht schicksalhaft über die Patienten hereinbricht. Konsequenzen dieser klaren Botschaft seien bislang allenfalls in Ansätzen zu erkennen. Fuchs äußerte zudem auch seinen Unmut über die von den KVen abgewinkten Gebührenordnungen, die eine zielführende allergologische Diagnostik verhindern würden. Als einzige Methode zum objektiven Nachweis einer Kontaktallergiegilt die Epikutantestung. Ausuferende Auflagen der Kontrollbehörden blockierten zudem die Diagnostik neuer und alter Kontaktallergene.

Die Folgen der (gesundheits-)politischen Gleichgültigkeit gegenüber der Problematik Kontaktallergie sind vielfältig. Nur ein Beispiel: Viele Duschgels und Shampoos, aber auch Haushaltsartikel wie Klarspüler und Flüssigseifen enthalten Methylisothiazolinone. Für die meisten Zeitgenossen dürfte der unbewusste Kontakt mit bekannten, aber offenbar verkehrsfähigen Kontaktallergenen unvermeidbar sein.

Dr. Ludger Riem

AllergoCompact: Dermatologie - Allergologie aktuell (Update Kontaktallergie) 